Neurosurg Focus 29 (3):E2, 2010

\title{
The pathogenetic features of cerebral cavernous malformations: a comprehensive review with therapeutic implications
}

\author{
Khaled M. Krisht, M.D., ${ }^{1}$ Kevin J. Whitehead, M.D., ${ }^{2}$ Toba Niazi, M.D., ${ }^{1}$ \\ and William T. Couldwell, M.D., PH.D. ${ }^{1}$ \\ ${ }^{I}$ Department of Neurosurgery and ${ }^{2}$ Division of Cardiology, Department of Medicine, University of Utah, Salt \\ Lake City, Utah
}

\begin{abstract}
Cerebral cavernous malformations (CCMs) are common vascular lesions of the CNS that may lead to seizures, focal neurological deficits, and fatal hemorrhagic stroke. Human genetic studies have identified 3 genes associated with CCM, and biochemical and molecular studies in mice have elucidated signaling pathways with important therapeutic implications. In this review, the authors shed light on the 3 discovered $C C M$ genes as well as their protein products, with particular emphasis on their signal transduction pathways and their interaction with one another. Close focus is directed at mice model studies involving the $\mathrm{Ccm} 2$ gene product signaling pathway, revealing an important role for the use of simvastatin or other RhoA inhibitors as a therapeutic modality in the treatment of CCM. The remaining challenges to creating a more faithful CCM animal model as well as future clinical and research implications are reviewed. (DOI: 10.3171/2010.6.FOCUS10135)
\end{abstract}

\section{KEY WoRds • cerebral cavernous malformation • CCM gene • signal transduction pathway $\quad \bullet \quad$ simvastatin}

$\mathrm{C}$ EREBRAL cavernous malformations are vascular lesions of the CNS that consist of a group of dilated, thin-walled, leaky capillaries with no intervening pia mater. This cluster of vessels or "caverns" is surrounded by a connective tissue matrix distinct from the surrounding pia mater. They are usually leaky, leaving a hemosiderin-laden rim circumferentially around the lesion that is easily detectable on T2-weighted echo-gradient MR imaging. Should the CM hemorrhage, the patient

Abbreviations used in this paper: $\mathrm{CCM}=$ cerebral cavernous malformation; GTP = guanosine triphosphate; HMVEC = human microvascular endothelial cell; HUVEC = human umbilical vein endothelial cell; ICAP1- $\alpha=$ integrin cytoplasmic domain-associated protein $1 \alpha$ JNK = c-jun N-terminal kinase; KRIT1 = Krev interaction trapped 1 ; LOH $=$ loss of heterozygosity; MAPK = mitogen-activated protein kinase; MEKK3 = mitogen-activated protein kinase kinase kinase 3; MKK3 = mitogen-activated protein kinase kinase 3 ; PDCD10 = programmed cell death 10 ; $\mathrm{PTB}=$ phosphotyrosine binding; siRNA = small interfering RNA; VEGF = vascular endothelial growth factor. may experience seizures or may sustain focal neurological deficits from either a stroke or focal irritation. ${ }^{19}$ Apart from the usual intracerebral location, CMs have also been observed in the spinal cord and retina, and as hyperkeratotic cutaneous capillary-venous malformations on the skin. The prevalence of CCMs in the general population is approximately $0.5 \%$, based on MR imaging and necropsy studies of large cohorts of patients. ${ }^{9,15}$ The clinical prevalence, however, is much lower, because only $25 \%$ of individuals are symptomatic. Symptoms usually develop between the 3rd and 5th decades of life..$^{15}$ The incidence of CCM is estimated at 0.2 to 0.4 lesions per patient-year. ${ }^{6,16}$ The diameter of the lesions ranges from a few millimeters to several centimeters. Histologically, CCMs have poorly formed tight junctions between adjacent endothelial cells, with gaps often noted between individual cells. Pericytes, the precursors to smooth muscle cells, are scant. In addition, no astrocytic foot processes and no normal nervous tissues are present within the lesion. ${ }^{4,19}$ 
Both sporadic and familial forms of CCM have been identified. The familial form of CCM follows an autosomal dominant form of inheritance, with incomplete penetrance and variable expression. The proportion of familial cases is estimated at 50\% among Hispanic-American patients of Mexican descent, and seems to be less in other populations. ${ }^{12,13} \mathrm{With}$ familial forms, multiple cerebral lesions are often noted (Fig. 1), whereas the majority of sporadic cases comprise a single CCM. By linkage and $\mathrm{LOH}$ analysis, 3 genetic loci have been implicated on chromosomal arms 7q (CCMI), 7p (CCM2), and $3 \mathrm{q}$ $(C C M 3){ }^{8,13}$ In this review, we describe the 3 aforementioned genes that have been implicated in CCM. We then focus our attention on $C C M 2$, with particular consideration of its molecular and pathophysiological implications and its clinical application in the treatment of CCM.

\section{The Ccm1 Gene}

The $C \mathrm{cml}$ gene, KRITl, was the first gene involved in CCM to be identified. It encodes a protein containing known protein-protein interaction domains. Using linkage analysis, a mutation in $C \mathrm{cml}$ was found in approximately $40 \%$ of familial cases. ${ }^{12}$ Despite the vascular nature of CCMs, KRIT1 mRNA and protein have been detected in astrocytes, neurons, and various epithelial cells during embryogenesis; however, only protein and not mRNA was detected in vascular endothelial cells

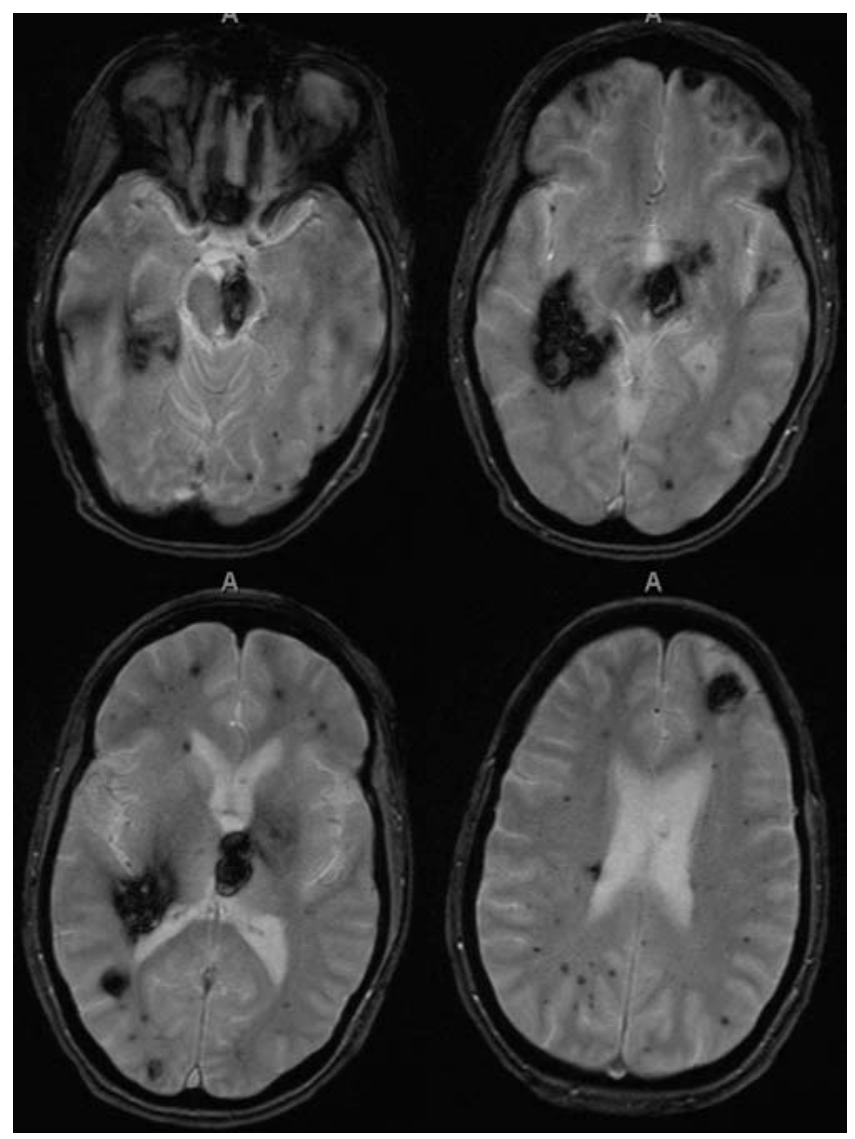

FIG. 1. Axial gradient-recalled echo MR images obtained in a 52year-old woman with familial CMs. This patient had multiple foci of gradient blooming artifact. during early angiogenesis. Murine embryos lacking the $\mathrm{Ccml}$ gene suggested an important role for KRIT1 in arterial morphogenesis and identity. The homozygous mutant embryos died in midgestation with vascular defects, including narrowed branchial arch arteries and decreased expression of artery-specific markers. ${ }^{6,19}$

KRIT1 interacts with ICAP1- $\alpha .{ }^{4}$ The latter is known to participate in integrin $\beta 1-$ mediated cell adhesion and migration. The interaction of integrin $\beta 1$ and KRIT1 with ICAP1- $\alpha$ occurs through an NPxY motif/PTB domain. ICAP1- $\alpha$ and KRIT1 have a functional nuclear localization sequence with the capacity to shuttle back and forth from cytoplasm to nucleus. Moreover, ICAP1- $\alpha$ is able to sequester KRIT1 in the nucleus. It seems, therefore, that KRIT1 acts as an intracellular signaling molecule through extracellular adhesion signals that are important for the activation of cellular differentiation and that help determine arterial identity., ${ }^{4,6,12,13,19}$

\section{The $\mathrm{Ccm} 2$ Gene}

The malcavernin (MGC4607) Ccm2 gene was discovered through LOH mapping and sequencing of positional candidate genes. As in Ccm1, in situ hybridization studies have shown $\mathrm{Ccm} 2 \mathrm{mRNA}$ expression in neurons and astrocytes as well as transient expression in meningeal and parenchymal cerebral vessels. ${ }^{7,11,16}$ In further molecular studies, it was noted that malcavernin contains a PTB domain similar to that of ICAP1- $\alpha$, suggesting an interaction between KRIT1 and malcavernin and, therefore, a common functional pathway. The similar phenotypic perturbation (failure of angiogenesis and vascular arrest during the embryonic stage) evident with $\mathrm{Ccm} 1$ and $\mathrm{Ccm} 2$ knockout mice further supports a common pathway involving the protein products of the respective genes. ${ }^{13} \mathrm{~A}$ working molecular model (Fig. 2) based on molecular genetic studies of CCM1 and CCM2 protein interaction and signaling proposes that CCM2 acts as a scaffold for Rac/ MEKK3/MKK3 for downstream p38 MAPK signaling. An RNA interference knockdown study has shown that malcavernin and MEKK3 were both required for activation of p38 in response to sorbitol-induced hyperosmotic stress. ${ }^{13}$ This points to a possible interaction between CCM2 and MEKK3. In addition, CCM2 heterozygous mice were shown to have significantly decreased levels of phosphorylated p38. Zawistowski et al. ${ }^{19}$ were also able to demonstrate that CCM1 can be detected with MEKK3 as a multiprotein complex involving CCM1 and CCM2 proteins. Therefore, a function of the CCM1/2 complex may be to organize and regulate p38 MAPK signaling. In their model, Zawistowski et al. proposed that ICAP1- $\alpha$ may tether CCM1 in the nucleus until a stimulus (osmotic stress/inflammation) is present, at which time CCM2 functions as a cytoplasmic anchor and recruits CCM1 to a signaling complex for integrin- and p38 MAPK-associated functions. The dynamics of the CCM1 cytoplasmic and nuclear localization suggests that this function is highly coordinated through compartmentalization.

\section{The Ccm3 Gene}

Bergametti and coworkers ${ }^{2}$ used $\mathrm{LOH}$ mapping to identify the $C \mathrm{~cm} 3$ gene. The $C \mathrm{~cm} 3$ gene, PDCD10, was 


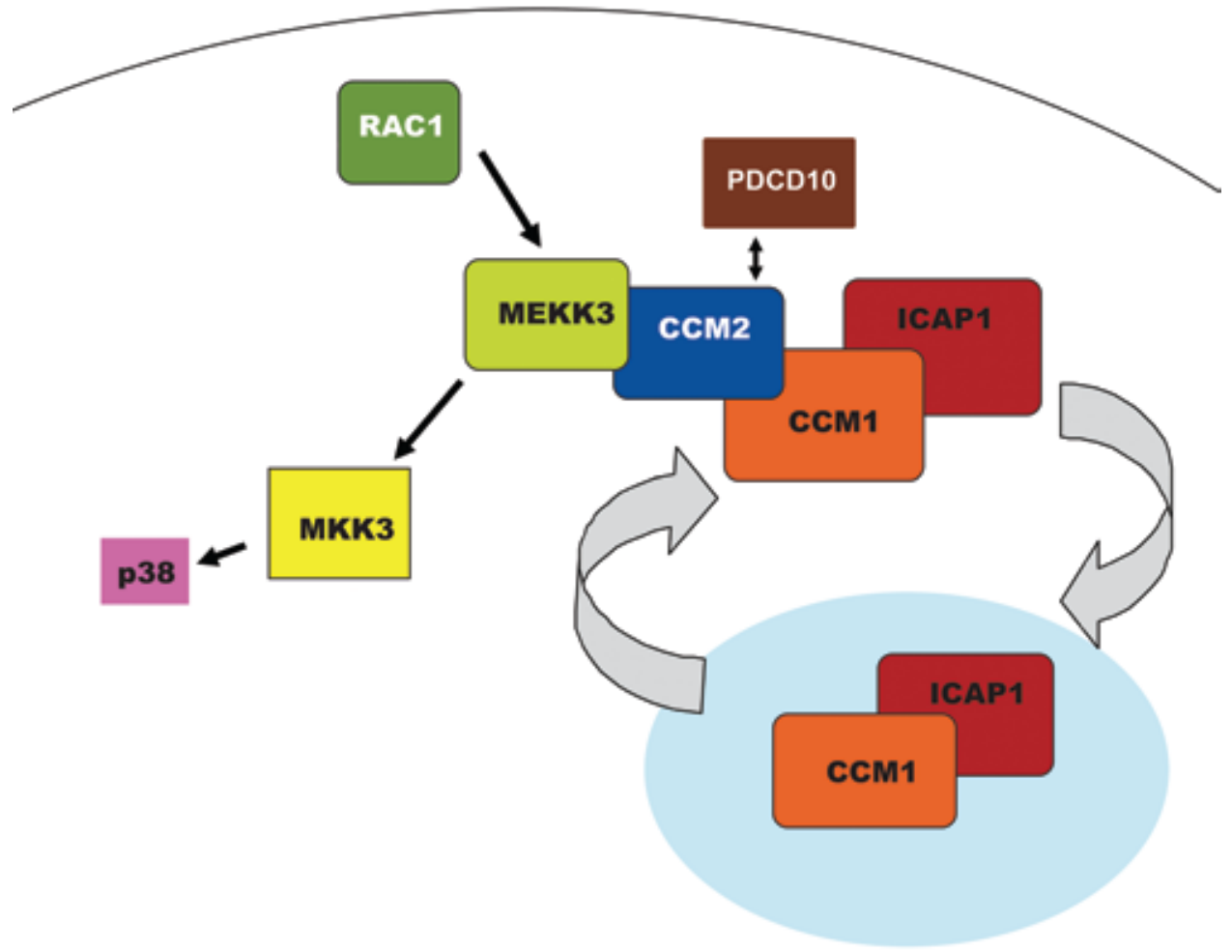

FIG. 2. Schematic presentation of molecular pathways involving the CCM proteins.

found to be ubiquitously expressed on the basis of Northern blot analysis. As with $\mathrm{Ccm} 1$ and $\mathrm{Ccm} 2$, in situ hybridization showed $\mathrm{Ccm} 3 \mathrm{mRNA}$ expression in neuronal cells at both adult and embryonic stages. In addition, its expression overlapped with that of $\mathrm{Ccm} 2$ in meningeal and parenchymal cerebral vessels. ${ }^{10,13}$ Interestingly, its expression was found to be upregulated in the human myeloid cell line TF-1 on induction of apoptosis. Because the expression of CCM3 seems to be very similar to that of CCM1 and CCM2, with overlapping mRNA patterns, and because PDCD10 has been shown to bind Krit1 and malcavernin, it most likely has a closely linked function. The endothelial and/or smooth muscle cell nature of vascular cells expressing Ccm 3 mRNA as well as the venous and/or arterial nature of the cerebral vessels expressing $\mathrm{Ccm} 3$ mRNA remain to be identified with antibodies specific for PDCD $10 .{ }^{10}$ Currently, several ongoing studies are examining expression patterns of CCM3. For further information, there are several recently published articles highlighting the cellular context of the various CCM protein actions, including a working model for CCM3., ${ }^{4,514}$

\section{From Bench to Bedside}

In an attempt to bridge the gap between bench work and the bedside, a number of studies have used animal models to begin to address the role of abnormal biochemical signaling pathways in states of CCM protein deficiencies. Whitehead et al. ${ }^{18}$ focused on the $C \mathrm{~cm} 2$ gene by studying mice with a gene trap mutation of $\mathrm{Ccm} 2$, and developing mice with tissue-specific, conditional mutations of $\mathrm{Ccm} 2$. Mice with a complete loss of $\mathrm{Ccm} 2$ share a common phenotype with mice lacking Kritl. In both instances, the homozygous knockout mice die in midgestation, having failed to connect the beating heart to the aorta through the branchial arch arteries. Although endothelium is present in the expected location, these cells fail to form a proper lumen, and circulation is not established. Using the Cre-Lox system to direct the mutation of $\mathrm{Ccm} 2$ to specific tissues, it was shown that $C \mathrm{~cm} 2$ is required in the endothelium for normal vascular development.

Having established a rationale for studying the role of CCM2 in endothelial cells, the authors used siRNA to deplete HUVECs or HMVECs for cell biology and biochemical studies. ${ }^{18}$ The CCM2-depleted HUVECs formed fewer lumina with a smaller cross-sectional area when compared with control cells. These observations point to the essential role of $\mathrm{Ccm} 2$ in endothelial cells for directing vacuole formation and coalescence to help form the vascular lumen. ${ }^{18}$ According to the studies by Bayless and Davis ${ }^{1}$ on microtubule depolymerization, lumen formation is dependent on the cellular cytoskeleton. Furthermore, CCM2-deficient HMVECs had an increase in actin stress fibers, with less cortical actin at the cell periphery, which was correlated with decreased barrier function and increased permeability. Because GTPases in the Rho family are important regulators of the cellular cytoskeleton, these observations suggested increased activity of the GTPase RhoA. Whitehead et al.$^{18}$ also observed increased active (GTP-bound) RHOA in CCM2-depleted HMVECs compared with control cells and found that inhibition of RHOA signaling was able to rescue the stress fiber and permeability defects in CCM2-depleted cells (Fig. 3).

The CCM proteins have also been found to interact with MAPK pathways. Although CCM2 had previously 
K. M. Krisht et al.
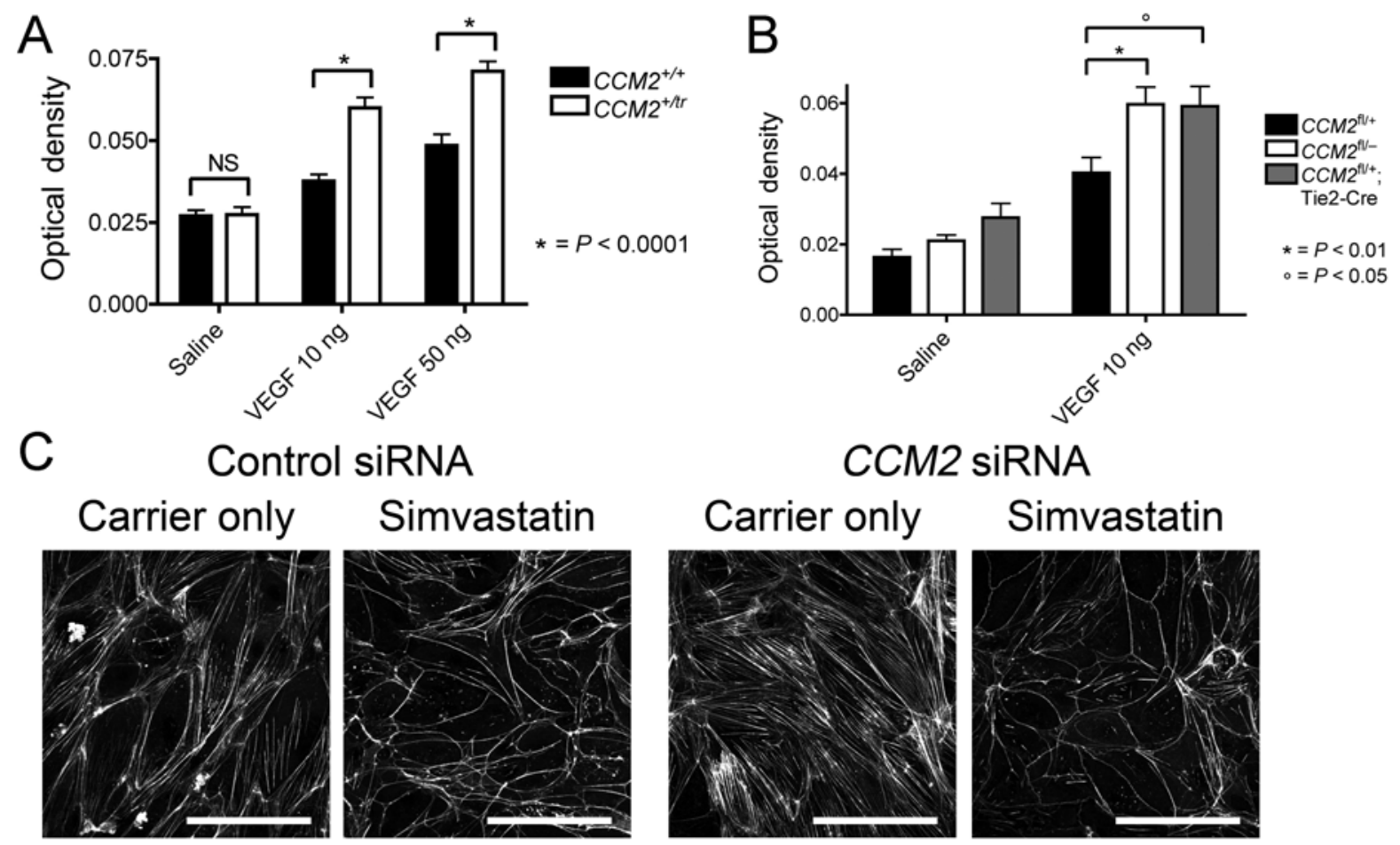

siRNA
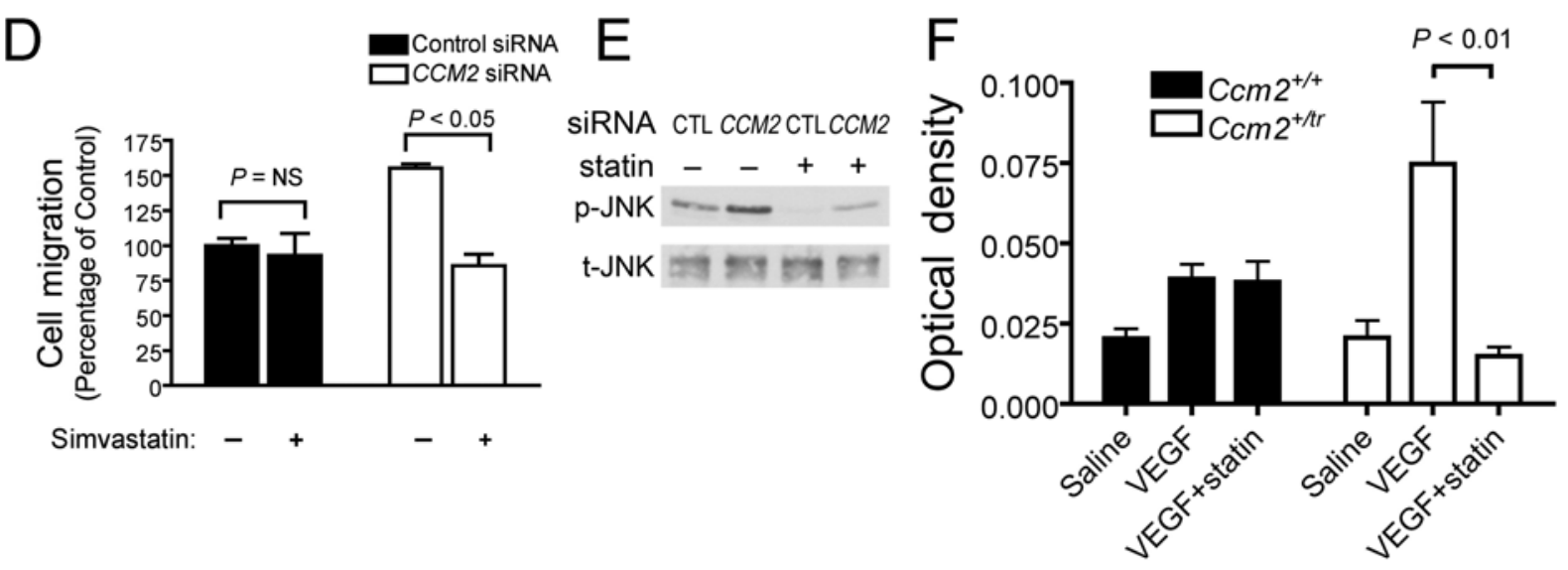

Fig. 3. Heterozygous $\mathrm{Ccm} 2^{+/ t r}$ mice have permeability defects that can be rescued by treatment with simvastatin. A: Bar graph showing spectrophotometric quantification of Evans blue extravasation in the Miles assay of dermal permeability in Ccm2 ${ }^{+/ t r}$ versus $\mathrm{Ccm}^{+++}$mice across a range of doses of VEGF compared with saline control. Five mice were studied for each genotype. B: Bar graph showing quantification of dermal permeability in mice with endothelial-specific heterozygosity for $\mathrm{Ccm} 2$ (Ccm2 $\left.{ }^{\text {Il+*}} ; \mathrm{Tie} 2-\mathrm{Cre}\right)$ compared with mice with both $\mathrm{Ccm} 2$ alleles intact $\left(\mathrm{Ccm} 2^{2 / /+}\right)$ and mice with complete $\mathrm{Ccm} 2$ heterozygosity $\left(\mathrm{Ccm} 2^{\mathrm{fl} /}\right)$. The authors studied $5 \mathrm{Ccm} 2^{i / /+}$ mice, $9 \mathrm{Ccm} 2^{f / /-}$ mice, and $10 \mathrm{Ccm} 2^{\mathrm{fll}+}$,Tie2-Cre mice. C: Phalloidin staining for cellular actin fibers after treatment with carrier or simvastatin. Results are representative of 3 independent experiments. Bar $=100 \mu \mathrm{m} . \quad$ D: Bar graph showing haptotactic migration of HMVECs to fibronectin after treatment with CCM2 or random control siRNA and treatment with either simvastatin or ethanol carrier. A minimum of 3 independent experiments were performed. E: Immunoblot for phosphorylated and total JNK in HMVECs treated with CCM2 or random control siRNA and treated with either simvastatin or ethanol carrier. Results are representative of 3 independent experiments. F: Bar graph showing quantification of Evans blue extravasation in the Miles assay in response to saline or VEGF after pretreatment with simvastatin or ethanol carrier. For both genotypes, 3 mice were used with control treatment and 4 mice with simvastatin treatment. Values are presented as the mean \pm SEM. Reprinted with permission from Macmillan Publishers Ltd: Nature Medicine (Whitehead KJ, Chan AC, Navankasattusas S, Koh W, London NR, Ling J, et al: The cerebral cavernous malformation signaling pathway promotes vascular integrity via Rho GTPases. Nat Med 15:177-184), copyright 2009. $C T L=$ control; $N S=$ not significant.

been shown to be important for p38 MAPK signaling in the cellular response to osmotic shock, Whitehead et al. ${ }^{18}$ found that a reduction of CCM2 increased the phosphorylation of JNK and its upstream kinases MKK4 and MKK7. Interestingly, Rho-kinase inhibitor decreased the phosphorylation level of JNK, pointing to a link between the GTPase and MAPK signaling. Therefore, a working molecular hypothesis is that loss of CCM2 leads to constitutive activation of RHOA and downstream activation of JNK that is also associated with cytoskeletal changes 


\section{Pathogenetic features of cerebral cavernous malformations}

that result in impaired lumen formation and vascular permeability. ${ }^{18}$

To test this hypothesis in vivo, we studied mice with heterozygous mutations in $\mathrm{Ccm} 2$ (the genotype equivalent of human disease). Although these mice appear normal and do not develop vascular lesions, increased vascular permeability was observed in the heterozygous mutants. Taking advantage of the ability of the cholesterol-lowering statin drugs to inhibit posttranslational lipid modifications of the Rho GTPases that are required for activity, Whitehead et al. ${ }^{18}$ showed that Rho inhibition by simvastatin was sufficient to stabilize the $\mathrm{Ccm} 2$-deficient endothelium in vivo (Fig. 3).

Subsequently, Stockton et al. ${ }^{17}$ showed that RhoA activation is also present in endothelial cells depleted of KRIT1, and that mice with heterozygous mutations of Kritl also have increased permeability. These authors demonstrated that an alternate strategy of Rho inhibition with the Rho-kinase inhibitor fasudil was also able to reverse the vascular stability defects of both $\mathrm{Ccm} 2$ and Krit1 deficiency in vivo. Together these studies suggest a central role for RhoA activation in the pathogenesis of CCM lesions in familial CCM associated with mutations in KRIT1 or CCM2.

Although a role for increased RhoA activation in vivo has not been shown for PDCD10, Borikova et al. ${ }^{3}$ recently demonstrated that, in addition to KRIT1 or CCM2, the loss of PDCD10 in endothelial cells also results in an increase in RhoA protein levels and activity. Taken together, these studies suggest the possibility that RhoA activation in endothelial cells of the CNS may represent a final common pathway for development of CCM lesions. Therapies directed at disrupting RhoA activity or downstream signaling such as the cholesterol-lowering statin family of drugs or the investigational agent fasudil are exciting candidates for medical therapy to stabilize CCM lesions and are worthy of further study.

\section{Future Prospects}

The working hypothesis of CCM onset is a "two-hit" hypothesis in which one germline mutant allele is inherited and the remaining somatic allele undergoes a spontaneous mutation, experiences an environmental insult such as osmotic stress, or has an inflammatory response that alters the stability of the endothelial cells that do not have the full CCM2 protector role as demonstrated with the in vivo VEGF studies. In a study by Zawistowski et al.,19 $\mathrm{Ccm} 2$ heterozygous p53 mutant mice showed an increased incidence as well as number of CCMs compared with control mice as a result of increased incidence of second-hit somatic mutations. The environmental as well as genetic second-hit model paves the way for future studies to unravel therapeutic means of reversing environmental insults as well as targeted gene therapy to add allelic stability. Taking advantage of the high rate of spontaneous mutations in mice lacking the tumor suppressor p53, Krit1 heterozygous knockouts have been mated onto a p53 knockout background in hopes of producing an adult viable mouse model of CCM, because the viable adult heterozygous mice develop no CCM lesions. ${ }^{6}$ Indeed, cerebral vascular lesions were observed in a large number of animals on this background, but the potential second-hit mutation was not localized. In addition, the mice had a shortened lifespan because of a high frequency of spontaneous tumors, making it difficult to study the natural history of CCM and its response to therapy ${ }^{4,19}$ Alternate means of inducing a second hit in an adult mouse without the confounding effects of the p53 mutation have yet to be reported, but it is hoped that such a strategy would create an animal model that closely resembles human CCM. Such a model could then be used to study the development of CCM lesions in vivo as well as the effect of therapeutic modalities.

Preliminary cellular and animal data suggest that statins may alter CCM biology. It is likely, given the widespread use of statins, that a considerable number of patients with CCMs have already been under observation while on this therapy. Further insight into the effect of statins on patients with known CCMs may be gained through retrospectively studying the clinical and radiological progression in those patients who are taking simvastatin for other medical indications. Between further preclinical studies of Rho inhibition in animal models and observational studies in humans, the goal is to establish a rationale for a double-blinded randomized controlled clinical trial to study the efficacy of statins on the course of CCM.

\section{Disclosure}

The authors report no conflict of interest concerning the materials or methods used in this study or the findings specified in this paper.

Author contributions to the study and manuscript preparation include the following. Conception and design: Couldwell. Acquisition of data: Krisht. Analysis and interpretation of data: Krisht, Niazi. Drafting the article: Krisht. Critically revising the article: Couldwell, Whitehead, Niazi. Reviewed final version of the manuscript and approved it for submission: Couldwell. Administrative/technical/ material support: Couldwell.

\section{References}

1. Bayless KJ, Davis GE: Microtubule depolymerization rapidly collapses capillary tube networks in vitro and angiogenic vessels in vivo through the small GTPase Rho. J Biol Chem 279:11686-11695, 2004

2. Bergametti F, Denier C, Labauge P, Arnoult M, Boetto S, Clanet M, et al: Mutations within the programmed cell death 10 gene cause cerebral cavernous malformations. Am J Hum Genet 76:42-51, 2005

3. Borikova AL, Dibble CF, Sciaky N, Welch CM, Abell AN, Bencharit S, et al: Rho kinase inhibition rescues the endothelial cell cerebral cavernous malformation phenotype. J Biol Chem 285:11760-11764, 2010

4. Chan AC, Li DY, Berg MJ, Whitehead KJ: Recent insights into cerebral cavernous malformations: animal models of CCM and the human phenotype. FEBS J 277:1076-1083, 2010

5. Faurobert E, Albiges-Rizo C: Recent insights into cerebral cavernous malformations: a complex jigsaw puzzle under construction. FEBS J 277:1084-1096, 2010

6. Leblanc GG, Golanov E, Awad IA, Young WL: Biology of vascular malformations of the brain. Stroke 40:e694-e702, 2009

7. Liquori CL, Berg MJ, Siegel AM, Huang E, Zawistowski JS, Stoffer T, et al: Mutations in a gene encoding a novel protein containing a phosphotyrosine-binding domain cause type 2 cerebral cavernous malformations. Am J Hum Genet 73:1459-1464, 2003 
8. Moriarity JL, Wetzel M, Clatterbuck RE, Javedan S, Sheppard JM, Hoenig-Rigamonti K, et al: The natural history of cavernous malformations: a prospective study of 68 patients. Neurosurgery 44:1166-1173, 1999

9. Otten P, Pizzolato GP, Rilliet B, Berney J: [131 cases of cavernous angioma (cavernomas) of the CNS, discovered by retrospective analysis of 24,535 autopsies.] Neurochirurgie 35:82-83, 128-131, 1989 (Fr)

10. Petit N, Blécon A, Denier C, Tournier-Lasserve E: Patterns of expression of the three cerebral cavernous malformation (CCM) genes during embryonic and postnatal brain development. Gene Expr Patterns 6:495-503, 2006

11. Plummer NW, Squire TL, Srinivasan S, Huang E, Zawistowski JS, Matsunami H, et al: Neuronal expression of the $\mathrm{Ccm} 2$ gene in a new mouse model of cerebral cavernous malformations. Mamm Genome 17:119-128, 2006

12. Reich P, Winkler J, Straube A, Steiger HJ, Peraud A: Molecular genetic investigations in the CCM1 gene in sporadic cerebral cavernomas. Neurology 60:1135-1138, 2003

13. Revencu N, Vikkula M: Cerebral cavernous malformation: new molecular and clinical insights. J Med Genet 43:716721,2006

14. Riant F, Bergametti F, Ayrignac X, Boulday G, TournierLasserve E: Recent insights into cerebral cavernous malformations: the molecular genetics of CCM. FEBS J 277:10701075,2010
15. Robinson JR, Awad IA, Little JR: Natural history of the cavernous angioma. J Neurosurg 75:709-714, 1991

16. Seker A, Pricola KL, Guclu B, Ozturk AK, Louvi A, Gunel M: CCM2 expression parallels that of CCM1. Stroke 37:518523,2006

17. Stockton RA, Shenkar R, Awad IA, Ginsberg MH: Cerebral cavernous malformations proteins inhibit Rho kinase to stabilize vascular integrity. J Exp Med 207:881-896, 2010

18. Whitehead KJ, Chan AC, Navankasattusas S, Koh W, London NR, Ling J, et al: The cerebral cavernous malformation signaling pathway promotes vascular integrity via Rho GTPases. Nat Med 15:177-184, 2009 (Erratum in Nat Med 15:462, 2009)

19. Zawistowski JS, Stalheim L, Uhlik MT, Abell AN, Ancrile $\mathrm{BB}$, Johnson GL, et al: CCM1 and CCM2 protein interactions in cell signaling: implications for cerebral cavernous malformations pathogenesis. Hum Mol Genet 14:2521-2531, 2005

Manuscript submitted May 14, 2010.

Accepted June 10, 2010.

Address correspondence to: William T. Couldwell, M.D., Ph.D., Department of Neurosurgery, University of Utah, 175 North Medical Drive East, Salt Lake City, Utah 84132. email: neuropub@hsc.utah. edu. 